\title{
Auditory Neuropathy/Dyssynchrony in Biotinidase Deficiency
}

\author{
Hossein Talebi ${ }^{1}$ and Omid Yaghini ${ }^{2}$ \\ ${ }^{1}$ Department of Audiology, Faculty of Rehabilitation, Communication Disorders Research Center, \\ Isfahan University of Medical Sciences, Isfahan, \\ ${ }^{2}$ Department of Pediatrics, School of Medicine, Growth and Development Research Center, Isfahan University of Medical Sciences, \\ Isfahan, Iran
}

Received February 18, 2016

Revised March 12, 2016

Accepted March 31, 2016

\author{
Address for correspondence \\ Hossein Talebi, $\mathrm{PhD}$ \\ Department of Audiology, \\ Faculty of Rehabilitation, \\ Communication Disorders \\ Research Center, Isfahan University \\ of Medical Sciences, \\ Hazarjarib Avenue, \\ Isfahan 81746-73461, Iran \\ Tel +983136693089 \\ Fax +983136693089 \\ E-mail ht6023@gmail.com
}

Biotinidase deficiency is a disorder inherited autosomal recessively showing evidence of hearing loss and optic atrophy in addition to seizures, hypotonia, and ataxia. In the present study, a 2-year-old boy with Biotinidase deficiency is presented in which clinical symptoms have been reported with auditory neuropathy/auditory dyssynchrony (AN/AD). In this case, transient-evoked otoacoustic emissions showed bilaterally normal responses representing normal function of outer hair cells. In contrast, acoustic reflex test showed absent reflexes bilaterally, and visual reinforcement audiometry and auditory brainstem responses indicated severe to profound hearing loss in both ears. These results suggest AN/AD in patients with Biotinidase deficiency.

J Audiol Otol 2016;20(1):53-54

KEY WORDS: Biotinidase deficiency · Auditory neuropathy/auditory dyssynchrony · TEOAEs · ABRs.

\section{Introduction}

Biotinidase deficiency is a disorder inherited as a recessive autosomal trait, with infantile onset. This disorder results in failure to recycle the vitamin, biotin [1]. The clinical symptoms of this disorder in untreated individuals with profound enzyme deficiency $(<10 \%$ of mean normal serum enzyme activity) include seizures, hypotonia, ataxia, breathing problems, alopecia, skin rash, hearing loss, vision atrophy, and developmental delay [2].

In this study, we show a case with possible auditory neuropathy/auditory dyssynchrony (AN/AD) caused by Biotinidase deficiency.

\section{Case Report}

T.J was a 2-year-old boy on admission that referred with his

This is an Open Access article distributed under the terms of the Creative Commons Attribution Non-Commercial License (http://creativecommons. org/licenses/by-nc/3.0/) which permits unrestricted non-commercial use, distribution, and reproduction in any medium, provided the original work is properly cited. parents from pediatric hospital to audiology clinic of Isfahan faculty of rehabilitation in May 2014. He was born at term to Iranian couple who were first-degree cousins. This study was carried out in accordance with the Helsinki Declaration. It is indicated that written informed consent was obtained from his parents. The pregnancy was without any problems. Delivery was performed normally in the hospital. There were not any perinatal and neonatal issues for this case. His parents and his older brother had abnormal vision.

Neurological examination indicated severe axial hypotonia, seizures, and ataxia. His visual system showed normal function evaluated by an ophthalmologist. Physical examination showed a slight conjunctivitis.

\section{Audiologic assessments}

In the present case, a comprehensive audiologic case history has been taken from his parents. Otoscopic inspection showed normal external ear canal and tympanic membrane without any middle ear effusion. Acoustic Immittance testing (tympanometry and acoustic reflex test) demonstrated normal tympanograms and absent ipsilateral and contralateral acoustic reflexes bilaterally. Visual reinforcement audiome- 
try was conducted in audiometric frequencies. The results indicated bilateral severe to profound sensorineural hearing loss. In addition, speech and language pathologist evaluated the patient's receptive and expressive speech and language skills and showed abnormalities and significant delay of speech perception and production in comparison to normal children. This case had a severe delay in speech development. He could not produce any words at 2-years-old. This problem is related to late diagnosis and treatment of hearing loss.

Auditory brainstem responses were recorded with 3 electrodes array in which non-inverting electrode was placed on the forehead, inverting electrode on the test ear mastoid and ground electrode on the non-test ear mastoid. The stimulus parameters were as follows: click duration $0.1 \mathrm{~ms}$, repetition rate $11.1 / \mathrm{s}$, sweep count 2,048, analysis time window of $10 \mathrm{~ms}$, low frequency cut-off $10 \mathrm{~Hz}$ and high frequency cut-off 3,000 $\mathrm{Hz}$, rarefaction and condensation polarities respectively presented via insert phones at different intensities for threshold estimation. The results showed complete absent responses at high intensity levels (in $\mathrm{dB} \mathrm{nHL}$ ) bilaterally indicating possibly severe to profound sensorineural hearing loss.

Finally, we conducted transient evoked otoacoustic emissions (TEOAEs) for both ears to assess the cochlear response. Otoacoustic emissions are responses emitted spontaneously or evoked by stimulus from the cochlea (outer hair cells). These responses show the outer hair cells, function [3]. In the present study, TEOAEs showed the normal function of the outer hair cells. This finding in addition to the above mentioned results indicated that the auditory system impairment could be occurred as a consequence of disturbed input from the inner hair cells, abnormal synaptic function, or peripheral neural pathology $[4,5]$. In other words, all of the responses of the patient showed the symptoms of AN/AD caused by Biotinidase deficiency.

\section{Discussion}

Sensorineural hearing loss in Biotinidase deficiency is shown in as many as $75 \%$ of individuals with symptoms.
This impairment and some other symptoms such as visual atrophy, and developmental delay are not usually reversible [1]. AN/AD describes patients who demonstrate intact outer hair cell function/active cochlear processes shown by OAEs and poor (complete absent) eight nerve/brainstem responses. Therefore, the site of origin for AN/AD is between the cochlea proper and the brainstem, with possible causes as injury to the synaptic junctions of the inner hair cells and/or the dendrites of the auditory nerve that receive neurotransmitters released by the inner hair cells, injury to the spiral ganglion, and/or damage to axons of cranial nerve VIII [4-6]. Further evidence of effects on neural function is demonstrated by generally absent or sometimes elevated middle-ear muscle reflexes [6]. Most AN/AD patients show bilateral symptoms. These patients show delayed or impaired development of speech and language $[4,5]$.

In this study, we present an infant with Biotinidase deficiency who showed evidence of AN/AD. It could be suggested that Biotinidase deficiency possibly results in any impairment in neural synchronization of auditory system.

\section{Conflicts of interest}

The authors have no financial conflicts of interest.

\section{REFERENCES}

1) Wolf B, Spencer R, Gleason T. Hearing loss is a common feature of symptomatic children with profound biotinidase deficiency. J Pediatr 2002;140:242-6.

2) Wolf B, Grier RE, Allen RJ, Goodman SI, Kien CL, Parker WD, et al. Phenotypic variation in biotinidase deficiency. J Pediatr 1983; 103:233-7.

3) Kemp DT, Brown AM. A comparison of mechanical nonlinearities in the cochleae of man and gerbil from ear canal measurements. In: Klinke R, Hartmann R, editors. Hearing-Physiological Bases and Psychophysics. Berlin: Springer-Verlag;1983. p.82-8.

4) Hood LJ. Auditory neuropathy: what is it and what can we do about it. Hear J 1998;51:10-8.

5) Starr A, Picton TW, Sininger Y, Hood LJ, Berlin CI. Auditory neuropathy. Brain 1996;119 (Pt 3):741-53.

6) Berlin CI, Hood LJ, Morlet T, Wilensky D, St John P, Montgomery $\mathrm{E}$, et al. Absent or elevated middle ear muscle reflexes in the presence of normal otoacoustic emissions: a universal finding in 136 cases of auditory neuropathy/dys-synchrony. J Am Acad Audiol 2005; 16:546-53. 